\title{
Discussion on the electromagnetic calorimeters of ATLAS and CMS
}

\author{
Martin Aleksa ${ }^{\mathrm{a}}$, Marcella Diemoz ${ }^{\mathrm{b}}$, on behalf of the ATLAS and CMS collaborations \\ ${ }^{a}$ CERN, Geneva 23, 1211 Geneva, Switzerland \\ ${ }^{b}$ INFN Roma, Piazzale Aldo Moro 2, 00185 Rome, Italy
}

\begin{abstract}
This document summarizes a discussion on the electromagnetic calorimeters of ATLAS and CMS, two experiments at the CERN Large Hadron Collider (LHC), that took place at the $13^{\text {th }}$ Vienna Conference on Instrumentation in February 2013. During the discussion each electromagnetic calorimeter and its performance was described in response to ten questions chosen to cover a wide range from the design and construction of the calorimeters over the calibration and performance to their role in the discovery of the Higgs boson and upgrade plans.
\end{abstract}

Keywords: Electromagnetic calorimetry, LHC experiments, ATLAS, CMS

PACS: $29.40 . \mathrm{Vj}, 14.80 . \mathrm{Bn}$

\section{Introduction}

The ATLAS [1] and CMS [2] detectors are multipurpose particle physics experiments with forward-backward symmetric cylindrical geometry at the CERN Large Hadron Collider (LHC). Both experiments optimized the electromagnetic calorimeters according to the physics requirements given by a few benchmark channels such as the discovery of the Higgs boson (e.g. $\left.\mathrm{H} \rightarrow \gamma \gamma, \mathrm{H} \rightarrow \mathrm{ZZ}^{(*)} \rightarrow 4 \mathrm{e}^{ \pm}, \mathrm{H} \rightarrow \mathrm{WW}^{(*)} \rightarrow \mathrm{e} v e v\right)$ and the discovery of physics beyond the Standard Model (e.g. decays of heavy $Z$ ' and $W^{\prime}$ ' with masses up to $6 \mathrm{TeV}$ ). In order to realize the full physics potential of the LHC, the ATLAS and CMS electromagnetic calorimeters must be able to identify efficiently electrons and photons within a large energy range ( $5 \mathrm{GeV}$ to $5 \mathrm{TeV}$ ), and to measure their energies with high resolution and per-mill level linearity. Despite very different approaches of the two collaborations concerning the overall layout, the design and even the type of their detectors, the overall performance in many physics channels is very similar. An impressive example is the discovery of the Higgs boson in summer 2012, where both experiments showed almost identical significances of their signals over background (see [3] and [4]). In the following we will describe the two electromagnetic calorimeters in greater detail and report on their performance observed during the first three years of data taking.

\section{Layout of the electromagnetic calorimeters}

\subsection{ATLAS}

The ATLAS electromagnetic calorimeter is a lead-liquid argon (LAr) sampling calorimeter with accordion geometry. It is described in detail in [5] and [1]. It is divided into a barrel region, covering a pseudorapidity ${ }^{1}|\eta|<1.475$, and two end-

\footnotetext{
${ }^{1}$ The pseudorapidity $\eta$ is defined in terms of the polar angle $\theta$ measured from the beam line as $\eta=-\ln \tan (\theta / 2)$.
}

caps covering $1.35<|\eta|<3.2$. The width of the active LAr gaps is $2 \mathrm{~mm}$ in the barrel and ranges from $1.2 \mathrm{~mm}$ to $2.7 \mathrm{~mm}$ in the endcap. The calorimeter is segmented into three longitudinal layers for $|\eta|<2.5$. The first layer, around 4-5 radiation lengths thick, is finely segmented in the $\eta$ direction to provide $\gamma$ $\pi^{0}$ separation and photon direction measurement. The cell size in $\eta$ is for instance 0.003125 in the barrel region. The second layer collects most of the shower energy and has a granularity of $\Delta \eta \times \Delta \varphi=0.025 \times 0.025$ for $|\eta|<2.5$. The last layer is used to correct for leakage behind the electromagnetic calorimeter. All layers together correspond to more than 22 radiation lengths. In addition a thin presampler layer is located between the cryostat and the calorimeter, covering the region $|\eta|<1.8$. It is used to correct for fluctuations in the energy loss upstream the calorimeter. Due to the position of the central solenoid coil inside the LAr barrel calorimeter and the aluminum cryostat walls the amount of material traversed by particles before reaching the electromagnetic calorimeter typically ranges between 3 and 6 radiation lengths (apart from a small transition region with higher values) depending on the pseudorapidity.

\section{2. $C M S$}

The design of the CMS electromagnetic calorimeter has been driven by the idea to achieve a very precise measurement of high energy photons in a very short space. Indeed a compact homogeneous calorimeter operating inside the $3.8 \mathrm{~T}$ superconducting solenoid ( $6 \mathrm{~m}$ inner diameter) of the experiment has been optimized to detect a Higgs boson in the low mass interval $100-150 \mathrm{GeV}$ through the decay $\mathrm{H} \rightarrow \gamma \gamma$. The CMS electromagnetic calorimeter is composed of Lead Tungstate $\left(\mathrm{PbWO}_{4}\right.$, in the following PWO) scintillating crystals organized in a barrel (61200 crystals of approximately $\left.2.2 \times 2.2 \times 23 \mathrm{~cm}^{3}\right)$ covering the central pseudorapidity region $|\eta|<1.48$ and two end-caps (14648 crystals of approximately $3 \times 3 \times 22 \mathrm{~cm}^{3}$ ) which extend the coverage up to $|\eta|<3$. A pre-shower detector, based 
on silicon sensors, placed in front of the end-caps is meant to improve the $\gamma-\pi^{0}$ separation in this region. The light from the crystals is detected by avalanche photo diodes (APD) in the barrel and vacuum photo triodes (VPT) in the end-caps. The compactness and transversal granularity of the calorimeter $\Delta \eta \times \Delta \varphi=0.0175 \times 0.0175$ reflect the properties of PWO, a very dense $\left(\rho=8.28 \mathrm{~g} / \mathrm{cm}^{3}\right)$ scintillating material characterized by a small radiation length $(0.89 \mathrm{~cm})$ and a small Molière radius $(2.0 \mathrm{~cm})$. After an extensive R\&D phase, the PWO crystals produced are fast ( $80 \%$ of light is emitted within $25 \mathrm{~ns}$ ) and suffer a moderate loss of transparency [8] with radiation for fluences and dose rates originally foreseen at the LHC. These changes of signal necessitate a very stable transparency monitoring system in order to maintain the design energy resolution of $0.5 \%$ at high energies. With the whole calorimeter being inside the CMS solenoid, the material in front is only due to the tracker system, and ranges from 0.4 and 1.9 radiation lengths depending on the pseudorapidity. A detailed description of the CMS electromagnetic calorimeter can be found in [6] and [2]

\section{Construction challenges}

\section{1. $C M S$}

The stochastic term of a homogeneus calorimeter is typically small $\sim 2-3 \%$. In order to achieve an excellent energy resolution it is necessary to keep the constant term, which dominates at high energy, below $0.5 \%$. Key points are the overall quality of the crystals and the system stability. Each of the 75848 crystals was qualified by measuring dimensions, optical parameters, light output and uniformity of the light output along the main axes [7]. To ensure a uniform radiation tolerance throughout the detector, strict optical tests were identified and performed on all installed crystals; a sample was also irradiated to verify directly the production quality. To follow and correct the variation of the single channel response expected with LHC radiation doses/fluxes a fibre-distributed laser system was designed to monitor the transparency of each single crystal [9]. This system, tested and optimized during test beam campaigns and commissioning with cosmic rays, has been proven to be stable to better than $0.2 \%$ [10] over extended periods. The dependence of the system on temperature $\left(-4 \%\right.$ per ${ }^{o} \mathrm{C}$ in the barrel, less in the end-caps) requires a stabilization to better than $0.05^{\circ} \mathrm{C}$ [11] in the barrel and about $0.1^{\circ} \mathrm{C}$ in the end-caps. The APD gain (around 50) varies strongly with bias voltage $(-3.1 \% / \mathrm{V})$ making the development of a very stable power supply system mandatory [12]. Both these systems proved to be fully compliant to the demanding specifications during the first three years of LHC running.

\subsection{ATLAS}

In order to achieve the required electromagnetic energy resolution utmost precision of the detector is necessary. Any possible variations of absorber thickness and absorber or electrode positions immediately translate into local non-uniformities of the energy response and hence contribute to a resolution degradation of the measured invariant mass (e.g. $\mathrm{H} \rightarrow \gamma \gamma$ mass peak). The construction of the LAr electromagnetic accordion calorimeter is documented in [13] (barrel) and [14] (endcap). To illustrate the utmost care that was taken during production it is worth recalling that in order to reduce thickness variations the lead thickness of absorbers was precisely measured during construction by means of X-rays at the rolling factory. On top of, that detailed thickness maps (on a $5 \times 5 \mathrm{~cm}^{2}$ grid) with an accuracy of a few $\mu \mathrm{m}$ were obtained from an ultrasound measurement system. Since one calorimeter cell comprizes 4 electrodes located between 4 absorbers an optimization of the arrangement of consecutive absorbers was performed to minimize the impact on phi-uniformity. The effect of this optimization resulted in the reduction of the rms of the sliding average from $0.44 \%$ to $0.31 \%$ without any systematic trends in phi. The residual contribution to the constant term of the energy resolution was estimated to $0.19 \%$ [13].

\section{Operational challenges and trigger}

\subsection{ATLAS}

Operating a high precision electromagnetic calorimeter with 173312 read-out channels requires careful online and offline monitoring. During most of the 2011 and 2012 data taking periods over $99.9 \%$ of the electromagnetic read-out channels were operational. It prooved to be essential to find small problems before the processing of the data that starts 48 hours after recording. During that time for example noisy cells get masked or events with large scale coherent noise are rejected. Less than $0.5 \%$ of data had to be discarded due to high voltage trips (in average there was about 1 trip per 10 hours of stable beam). After all cuts in $201299.1 \%$ of the recorded LAr calorimeter data had good data quality, physics studies requiring good quality data of all ATLAS detectors used $95.8 \%$ of the recorded data.

The LAr calorimeter also delivers analog signals with tower energies $(\Delta \eta \times \Delta \varphi=0.1 \times 0.1)$ to the ATLAS L1 calorimeter trigger. The full granularity digital read-out is used for higher level trigger decisions. The lowest unprescaled single electron trigger in 2012 had a transverse energy $\left(E_{\mathrm{T}}\right)$ threshold of $24 \mathrm{GeV}$. The $\mathrm{H} \rightarrow \gamma \gamma$ data sample was recorded with a diphoton trigger which requires $35 \mathrm{GeV}$ and $25 \mathrm{GeV} E_{\mathrm{T}}$ thresholds for the leading and sub-leading photons, respectively. This trigger has a signal efficiency above $99 \%$ for events fulfilling the final event selection.

\section{2. $C M S$}

The calorimeter was installed in CMS with very few problematic channels. The current number of active channels is stable in time, around $99.1 \%, 98.5 \%$, and $97 \%$ in barrel, end-caps and preshower respectively. Some of the malfunctioning channels will be recovered during the first long shut down (LS1) of LHC. The operational reliability is very high: in 2011 and 2012 less than $1 \%$ downtime of CMS has been caused by the calorimeter, and more than $99 \%$ of data collected are good for analysis. This excellent performance is the result of careful design, an efficient online/offline set of monitoring tools and to a group of competent and highly dedicated people. 
The L1 electromagnetic trigger is based on the sum of transverse energy deposited in adjacent trigger towers (e.g. $5 \times 5$ matrices of crystals in the barrel). In 2011 the L1 single-photon trigger with an $\mathrm{E}_{T}$ threshold of $15 \mathrm{GeV}$ was run unprescaled, with an efficiency measured to be $99 \%$ for $E_{\mathrm{T}}=20 \mathrm{GeV}$, thus being fully efficient for the $\mathrm{H} \rightarrow \gamma \gamma$ channel (leading photon $\left.E_{\mathrm{T}}>35 \mathrm{GeV}\right)$. The $\mathrm{L} 1$ trigger efficiency was also very stable in time despite some issues that arose. For example, direct ionization in the APD silicon before the acceleration gap can produce apparent 'high energy' signals. These anomalous signals ("spikes") should be removed online at L1 otherwise they may cause a high spurious trigger rate and saturate the bandwidth assigned to electromagnetic objects [15]. Two characteristics are used to reject the spikes: transverse shower shape (spikes are typically seen in isolated single crystals, contrary to e.m. showers that can spread over 10-30 crystals) and timing (the scintillation time of PWO crystal ( $\sim 10 \mathrm{~ns})$ is absent so the spike pulse usually arrives 'early'). The flexibility of the front-end electronics allows a coarse shower-shape evaluation in the L1 trigger, allowing the rejection of about $96 \%$ of spikes whilst maintaining more than $99 \%$ trigger efficiency. The few anomalous signals that survive the L1 filter are eliminated at the subsequent trigger step (HLT) by applying a rejection based on the hit timing in the crystal (resolution $1 \mathrm{~ns}$ ) and a simple but effective shower-shape algorithm.

\section{Calibration}

\section{1. $C M S$}

Accurate calibration is critical for obtaining the best estimate of electron and photon energies. Given the small Molière radius $80 \%$ of the energy of the impinging e $\gamma$ is deposited in a single crystal (for central incidence). The reconstruction of the total energy implies the summation of energy deposits in a cluster of crystals. The energy may also be more or less spread in $\varphi$ due to the strong magnetic field, the non-negligible material in front of the calorimeter and whether the particle is an electron or a photon. Dedicated algorithms have been developed to reconstruct "superclusters" in order to measure the initial energy of the particle as precisely as possible.

$$
E_{\mathrm{e} / \gamma}=F_{\mathrm{e} / \gamma} G \sum_{x l} c_{x l} L(t)_{x l} A_{x l}
$$

The energy deposited in each crystal is given by the pulse amplitude $A_{x l}$, in ADC counts, multiplied by ADC-to-GeV conversion factor $G$, measured separately for the barrel (EB) and end-caps (EE), by the intercalibration coefficients $c_{x l}$ of the corresponding channel, and by $L(t)_{x l}$, a correction term due to radiation-induced channel response at time $t$. The calibrated preshower energy is added to the end-cap energy deposits. The factor $F_{\mathrm{e} / \gamma}$ represents the energy correction applied on the superclusters necessary to take into account the $\eta$ - and $\varphi$-dependent geometry and material effects as well as the fact that electrons and photons interact slightly differently in the calorimeter. The relative variation of the channel response, measured by the laser light injection, is shown in Fig. 1 as a function of time (irradiation) for different $\eta$ regions.

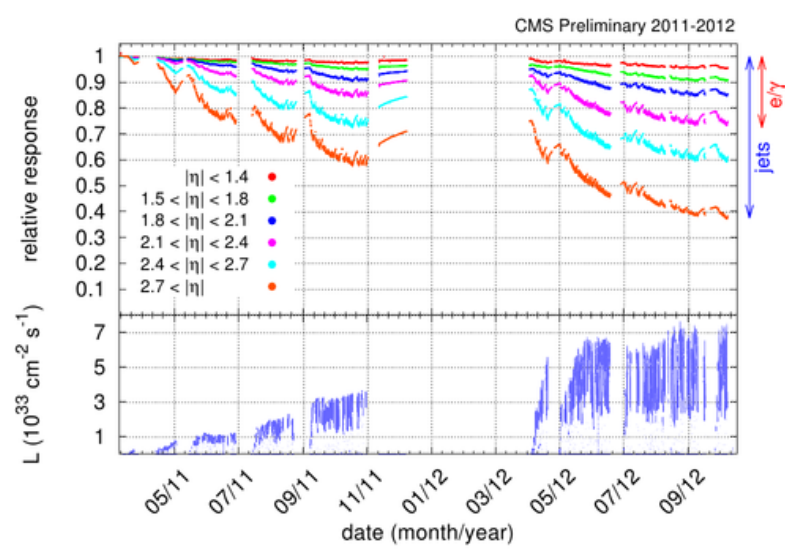

Figure 1: Relative response of the CMS ECAL to laser light during 2011 and 2012, normalized to data at the start of 2011. An average is shown for each pseudorapidity range. The bottom plot shows the corresponding instantaneous luminosity. A partial recovery of crystal transparency is observed during technical stops of the LHC and the low luminosity heavy-ion data-taking in December 2011.

Correction factors, to account for response changes, are calculated online and are available a few hours after having taken data. The corrected signal is restored and checked using the decay $\eta \rightarrow \gamma \gamma$. The laser system is itself monitored (by PN diodes) and is very stable in time, as shown in figure 2 . Once validated, the correction factors are inserted into a database and promptly-corrected data can be used for many physics analyses. More refined offline corrections are, on the other hand, necessary to reach the best performance of the calorimeter. The intercalibration between individual channels is obtained from collision data using different physics processes. The $\varphi$-symmetry method is based on the invariance of the energy flow around the beam axis in minimum bias events. This method is used to intercalibrate crystals at the same pseudorapidity (248 $\eta$ rings of crystals). The method suffers from any increase of single channel noise but profits from pile-up (more hits per crystal). It is the fastest method but its accuracy is limited by systematics, mainly due to the fact that the upstream material (e.g. tracker layers and support structure) is not uniformly distributed in $\varphi$. The $\pi^{0} / \eta$ method uses the mass constraints on photon pairs. Unconverted pairs of photons in $3 \times 3$ matrices of crystals are selected online as $\pi^{0} / \eta$ candidates. High statistics are used for single channel intercalibration in $\varphi$. The signal-to-noise for detecting/measuring the photons from these decays is sensitive to any increases in single-channel noise. Calibration at higher energies is performed through the use of single electrons from $\mathrm{W}$ decays. The ratio of the calorimeter energy $E$ to the tracker momentum $p$ is used to intercalibrate the average response of the $248 \eta$ rings. With the 2011/2012 luminosity the precision is still limited by statistics, but the same method can, in principle, be used for single channel intercalibration. It is an important tool to monitor the calorimeter response stability over a wide energy range. Inter-calibrations from the different methods are 
combined to give the overall inter-calibration coefficients. The present combined precision of the inter-calibration depends on $\eta$. In the central barrel $\eta \leq 1$ the precision is $0.5 \%$, rising to $0.7 \%$ in the highest $\eta$ region of the barrel. In the end-caps the precision ranges from $1 \%$ to $2.5 \%$ depending on the distribution of the material in front. The cluster energy correction $F_{e / \gamma}$ can be as high as a few percent in regions with a significant amount of material in front of the calorimeter, in regions of transition between calorimeter modules and is different for non-showering (cluster shape variable $R 9=E_{3 \times 3} / E_{S C} \geq 0.94$ where $E_{3 \times 3}$ is the energy sum in a matrix of 9 crystals and $E_{S C}$ is the energy reconstructed in the corresponding supercluster) and showering $\left(R 9=E_{3 \times 3} / E_{S C}<0.94\right)$ electrons. It is generated by a Monte-Carlo (MC) driven multivariate analysis (MVA) based on shower shape, shower location and event energy density (pile-up), and recovers energy leakage (gaps, cracks), energy loss before the calorimeter and unclustered energy. Work is ongoing to improve these corrections even further, particularly for the recovery of the energy leakage in module boundaries. The final step in the calibration procedure is the calculation of the global energy scale, described in detail in section 6.1. This is found by measuring di-electrons from $\mathrm{Z}$ decays and using the $\mathrm{Z}$ invariant-mass peak. The width of the peak is also a useful parameter for studying the calorimeter resolution, especially relevant for searches for particles around $100 \mathrm{GeV}$.

\subsection{ATLAS}

The calibration of the electron and photon energy scales can be seen as a three-step process: The cell-level calibration of the read-out electronics, the MC-based cluster level calibration and the in-situ calibration using invariant mass peaks of e.g. $Z \rightarrow$ ee (see Sec. 6). The concept of LAr calorimeter electronics calibration is to inject a well known exponential pulse as close as possible to the point where the ionization pulse is usually created in the detector and read it back with the normal front-end electronics. In that way electronics gain, pulse shapes and pedestals are extracted on a regular basis and updated in the database in case differences are detected (usual update frequency is once per month). Figure 3 shows the excellent stability of the electronics gain throughout the 2011 data taking. The rms of the distribution of the average gain deviations per front end board ${ }^{2}$ as a function of time is $2.56 \cdot 10^{-4}$.

In order to obtain energies of electrons and photons, the energies of cells in a region around a high energy deposit are summed into clusters (e.g. cluster size of $\Delta \eta \times \Delta \varphi=$ $0.075 \times 0.175$ for electrons and converted photons in the barrel). However, a simple sum of cell energies is not sufficient since the energy lost upstream, the energy outside the cluster and the energy behind the calorimeter (longitudinal leakage) needs to be corrected for. These corrections are done using pseudorapidity- energy- and shower-depth dependent calibration constants which are extracted from MC simulations (different constants for electrons, unconverted photons and converted photons). These constants do not depend on time nor

\footnotetext{
${ }^{2}$ electronics board processing signals of 128 calorimeter cells
}

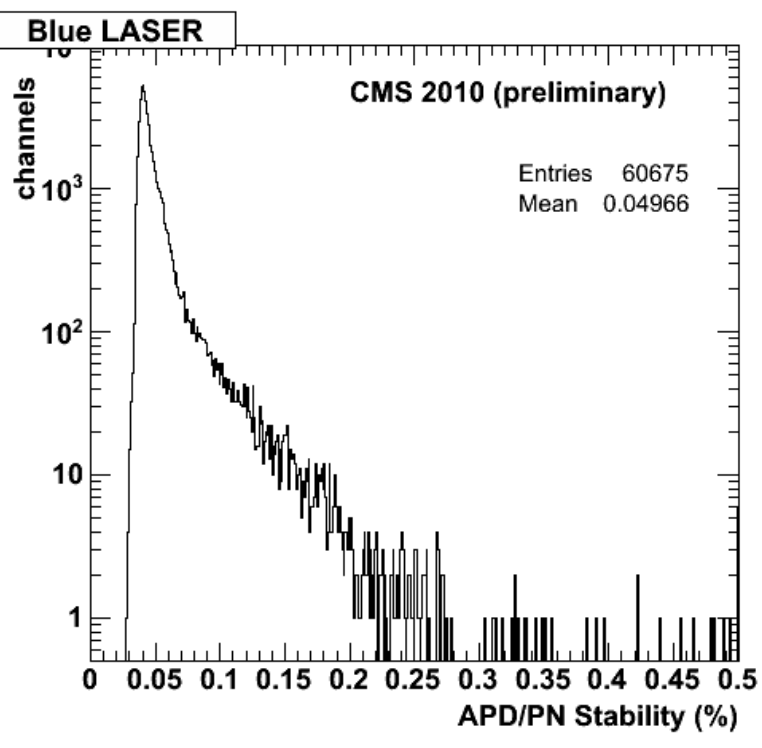

Figure 2: CMS laser system and electronic readout stability over a period of $350 \mathrm{~h}$, each single channel response to injected laser light in absence of irradiation is compared to a reference PN diode.

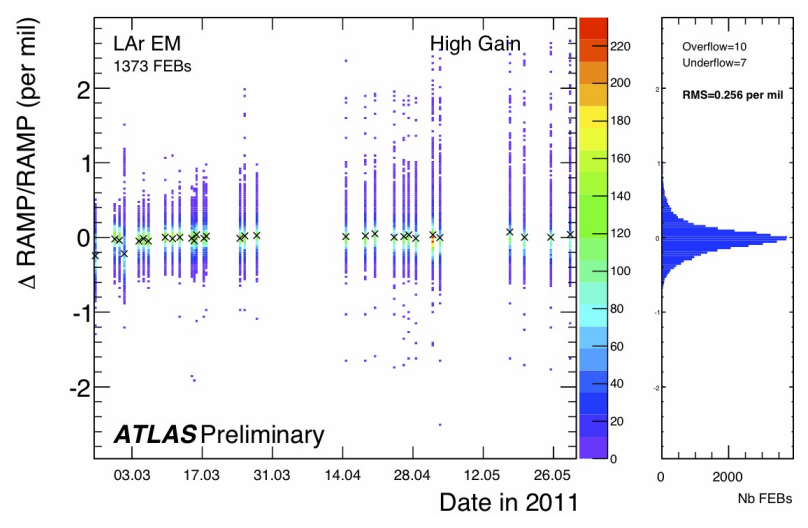

Figure 3: Stability of the ATLAS electronics gain during the year 2011 .

luminosity. It is obvious that an accurate MC description of the calorimeter and the material in front of the calorimeter is essential for this approach. Many data/MC comparisons have been done to verify and correct the MC description.

\section{Resolution and uniformity}

The calorimeter resolution is usually parameterized as

$$
\frac{\sigma_{E}}{E}=\frac{a}{\sqrt{E}} \oplus \frac{b}{E} \oplus c
$$

where $a$ is the stochastic term, $b$ the noise term and $c$ the constant term.

\subsection{ATLAS}

The resolution of the electromagnetic calorimeter as a function of energy was measured previously in testbeams [1], 
a stochastic term $a$ of $10 \% \sqrt{\mathrm{GeV}}$, a noise term $b$ around $200 \mathrm{MeV}$ and a constant term $c$ of around $0.2 \%$ was obtained for the barrel. The uniformity was measured in testbeams to be better than $0.5 \%$ for barrel modules and better than $0.6 \%$ for endcap modules [16]. It is very difficult to perform such measurements in-situ with collisions data. One way to probe the constant term $c$ is to compare the line shape of the $\mathrm{Z}$ between data and MC [17]. Assuming that the stochastic term $a$, that prevails for small energies, is well modeled by MC - an assumption that seems to be confirmed by the excellent data-MC agreement of the width of the $\mathrm{J} / \Psi$ line-shape - an effective constant term $c_{\text {eff }}$ can be extracted from the measured line-shape of the Z. The exact procedure is described in [17] and yields $c_{\text {eff }}=1.2 \% \pm 0.1 \%$ (stat) ${ }_{-0.6 \%}^{+0.5 \%}$ (syst) in the barrel $(|\eta|<1.37)$ and $c_{\text {eff }}=1.8 \% \pm 0.4 \%$ (stat) $\pm 0.4 \%$ (syst) in the precision region of the endcap $(1.52<|\eta|<2.5)$. Figure 4 shows the di-electron mass distributions for the barrel.

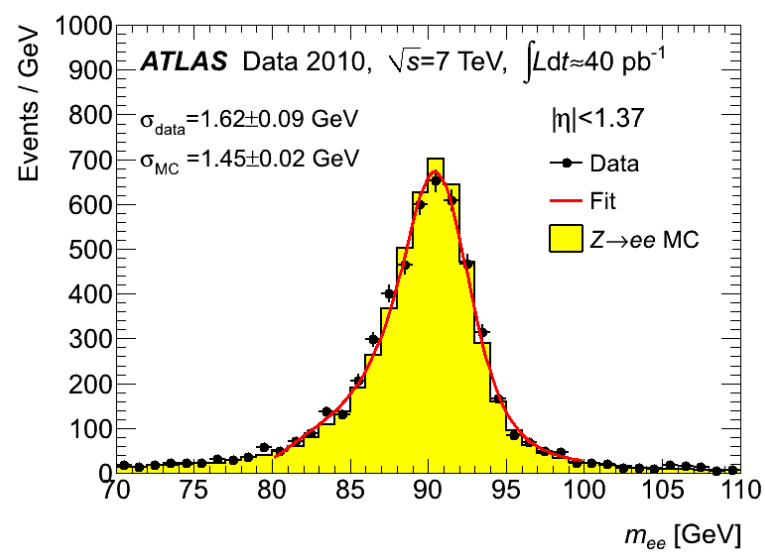

Figure 4: Reconstructed di-electron mass distributions for $\mathrm{Z} \rightarrow$ ee decays for $|\eta|<1.37$ after applying the baseline Zee calibration in ATLAS [17].

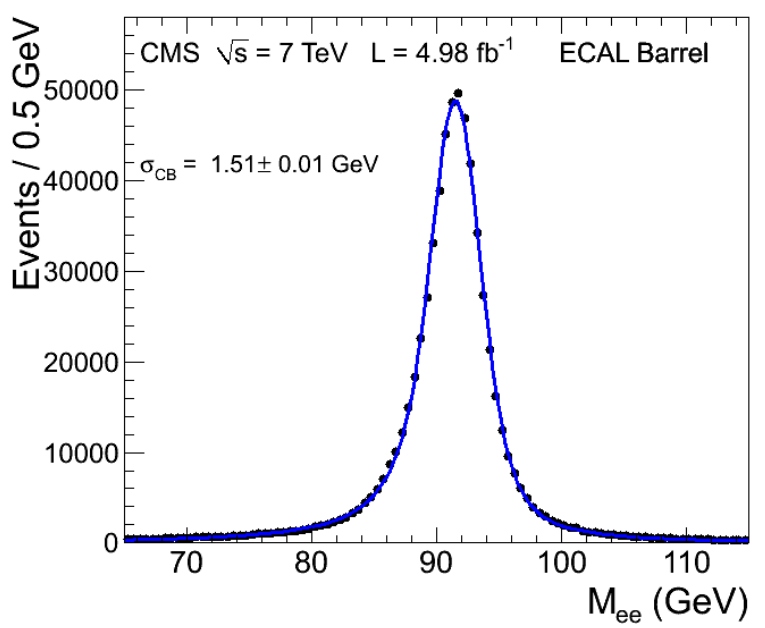

Figure 5: $\mathrm{Z} \rightarrow$ ee invariant mass distribution with both calibrated electrons in the CMS barrel [18].

\section{2. $C M S$}

The resolution of the electromagnetic calorimeter, as a function of energy, was measured in beam tests [19] in optimal conditions. A stochastic term $a$ of $2.8 \% \sqrt{\mathrm{GeV}}$, a noise of $128 \mathrm{MeV}$ for the sum of a $3 \times 3$ energy cluster and a constant term $c$ of $0.3 \%$ have been obtained for central crystal impact on several barrel modules. These figures, and the current precision on the intercalibration coefficients $c_{x l}$, which contributes to the constant term $c$, are injected as input to the MC simulation in order to describe the behaviour of the calorimeter. In-situ the resolution is estimated from the $\mathrm{Z}$ lineshape for showering and nonshowering electrons separately for barrel and end-caps. Figure 5 shows the inclusive $\mathrm{Z} \rightarrow \mathrm{e}^{+} \mathrm{e}^{-}$invariant mass distribution with both electrons in the barrel. The data are fitted with a convolution of a Breit-Wigner (parameters fixed to the nominal values $M_{Z}$ and $\Gamma_{Z}$ ) and a Crystal Ball (CB) function to model the experimental resolution. The width of the Gaussian term of the $\mathrm{CB}, \sigma_{C B}$, represents the calorimeter resolution and can be compared with $\mathrm{MC}$ expectation. The measured $\mathrm{Z}$ invariant mass resolution is related to the single electron resolution by a factor $\sqrt{2}$. The observed relative mass resolution is $1.6 \%$ in the barrel $(1.5 \mathrm{GeV} ; 1 \mathrm{GeV}$ if both electrons are non-showering) and $2.6 \%$ in the end-caps $(2.4 \mathrm{GeV})$. In both barrel and end-caps, for non-showering $R 9 \geq 0.94$ and showering $R 9<0.94$ electrons the experimental resolution is not perfectly reproduced by the simulation. As an example, the $\sigma_{C B}$ predicted by simulation for both electrons (all values of R9) in the barrel is $1.29 \mathrm{GeV}$. This discrepancy can be due to several small effects, which are not yet described with sufficient accuracy and that are under investigation. To make the MC reproduce experimental data an additional smearing is applied in bins of $\eta$ and $R 9$, to electron and photon energies. The smearing term is obtained by minimizing the difference between the observed $\mathrm{Z} \rightarrow$ ee lineshape in data and in the smeared MC [18] [20]

This approach, which takes into account the deterioration of the energy resolution with respect to the design one by introducing an additional constant term, might be pessimistic. The material upstream the calorimeter plays an important role (details of its distribution are difficult to be determined) and affects the resolution as an additional stocastic term. Further studies are ongoing to improve the detector description in the simulation.

\section{Energy scale and linearity}

\subsection{CMS}

The absolute scale $G$ is computed in a reference region of the calorimeter, separately for barrel and end-caps, where the effects of the upstream material have the lowest impact. The ADC-to-GeV conversion factor is calibrated using the $\mathrm{Z}$ lineshape by requiring that the fitted peak in data agrees with that of the MC simulation. The systematic uncertainty on $G$ is mainly due to the accuracy of the cluster energy corrections $F_{e}$ and in the 2011 data sample is estimated to be $0.4 \%$ in the barrel and $0.8 \%$ in the end-caps. Figure 6 shows a preliminary measurement of the electron energy scale as a function of $p_{T}$, which 
combines the tracker momentum and the calorimeter cluster energy measurements in a weighted average. The low $p_{T}$ region is dominated by the accuracy of the tracker momentum measurement.

\subsection{ATLAS}

The electron energy scale is extracted in situ as a function of pseudorapidity $\eta$ using the peak of the invariant mass distribution of $\mathrm{Z}$ candidates (see Fig. 4). Cross checks are done using the the ratio of the energy measured in the electromagnetic calorimeter over the momentum measured in the inner tracker $E / p$ (electrons from $\mathrm{W} \rightarrow \mathrm{e} v$ and $\mathrm{Z} \rightarrow \mathrm{ee}$ ) and the invariant mass distribution of $\mathrm{J} / \Psi \rightarrow$ ee (lower energy electrons than $Z)$ and $Z \rightarrow$ ee $\gamma$ for photons. The obtained electron energyscale correction factor $\alpha$ is determined as a function of pseudorapidity and corrects the energy scale by $\approx 1 \%$ in the barrel and up to $5 \%$ in the endcap. The residual systematic errors on the electron energy-scale are estimated to be $0.3 \%$ in the barrel and $\approx 1 \%$ in the endcap (including material uncertainty). The photon scale is derived from the electron energy-scale by adding MC-based corrections for both, converted and unconverted photons. Figure 7 shows the energy scale correction factor $\alpha$ as a function of energy (linearity of the energy scale). All measurements are within the attributed uncertainty. Eventually for precision measurements like e.g. the $\mathrm{W}$ mass a linearity at the level of few $10^{-4}$ is necessary.

\section{Measured stability in time and vs pile-up}

\subsection{ATLAS}

Together with a very stable LAr bath purity of below $300 \mathrm{ppb}$ and a very stable and uniform LAr bath temperature, the very stable ATLAS read-out electronics (see Sec. 4) guarantee the stability of the energy response of the electromagnetic calorimeter. Figure 8 shows the stability of the energy-scale for $|\eta|<2.5$ (transition region between barrel and endcap excluded) as a function of time during part of the year 2012 using $E / p$ of $\mathrm{W} \rightarrow \mathrm{e} v$ events and the position of the $\mathrm{Z} \rightarrow e e$ invariant mass peak. The measured variations have an rms smaller than $3.5 \cdot 10^{-4}$ which includes the (small) statistical errors of the individual points. Also no energy-scale variation as a function of pile-up is observed (see Fig. 9, rms smaller than $2 \cdot 10^{-4}$ ).

\section{2. $C M S$}

The stability in time of the calorimeter response is dominated by the accuracy of the laser correction and is measured using the $E / p$ ratio in $\mathrm{W} \rightarrow \mathrm{e} v$ events, as mentioned in section 4.1. Figure 10 shows that the calorimeter response to electromagnetic showers in the barrel during the 2011 data-taking is stable with an RMS of $0.12 \%$; in the end-caps the RMS is $0.35 \%$. The worse performance in the end-caps is probably due to a non perfect control of all the effects induced by the heavier irradiation in this higher $\eta$ region. The robustness of the energy scale determination was also studied and a specific MC-driven MVA algorithm developed to mitigate effects of pile-up. Figure 11

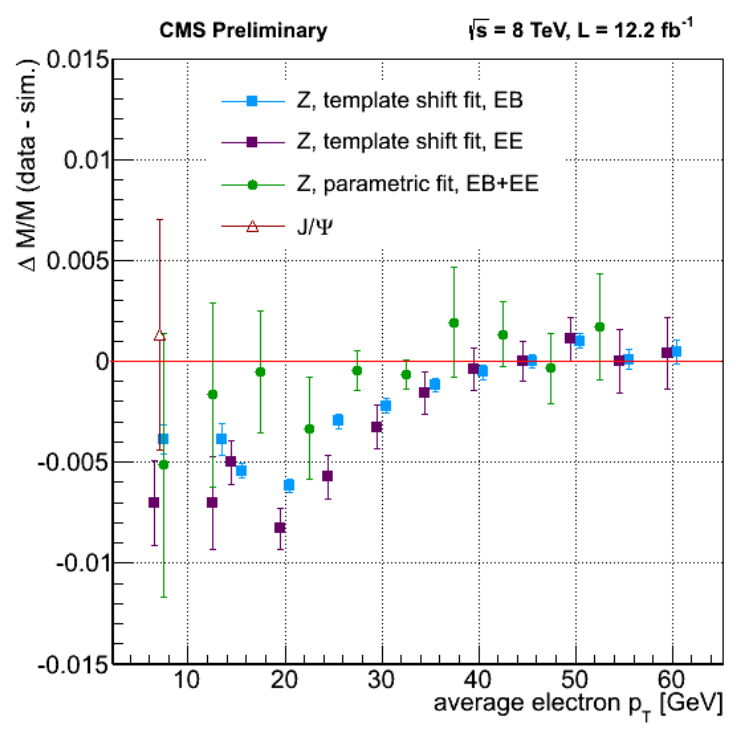

Figure 6: Relative difference between the dielectron mass scales in CMS data and simulation for $\mathrm{Z} \rightarrow$ ee and $\mathrm{J} / \Psi \rightarrow$ ee events as a function of the electron transverse momentum. Momentum (measured in the tracker) and energy (measured in the calorimeter) are combined in a weighted average. The low $p_{T}$ region is dominated by the tracker measurement.

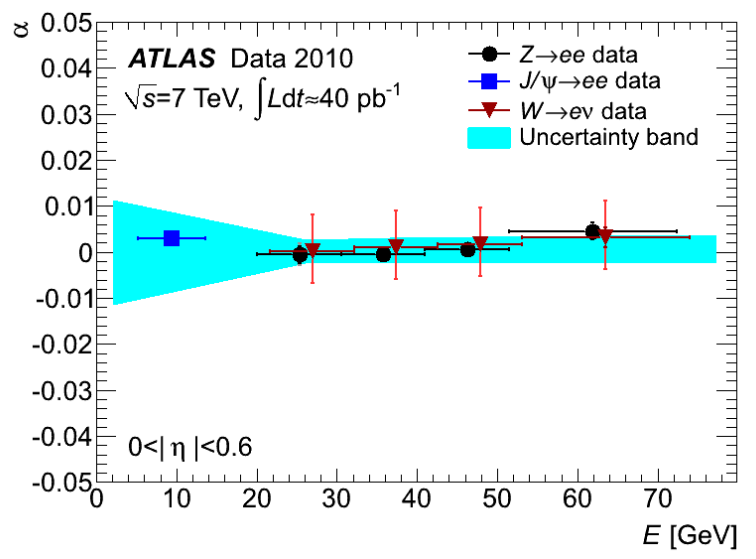

Figure 7: First in-situ measurement of the ATLAS energy scale linearity for $|\eta|<0.6$ [17].

shows the stability of the scale using the default reconstruction of $E / p$ and its slight dependence on the number of reconstructed vertices in the event (perfectly reproduced by MC); the pile-up corrected scale is stable with the number of vertices.

\section{Electron and photon identification efficiency}

\section{1. $C M S$}

The identification of electromagnetic objects in a crystal calorimeter is rather straightforword. In CMS the strong magnetic field, the material upstream and the effects of pile-up make life a bit more complicated. A set of variables are used to select electromagnetic objects. These include the shower width 


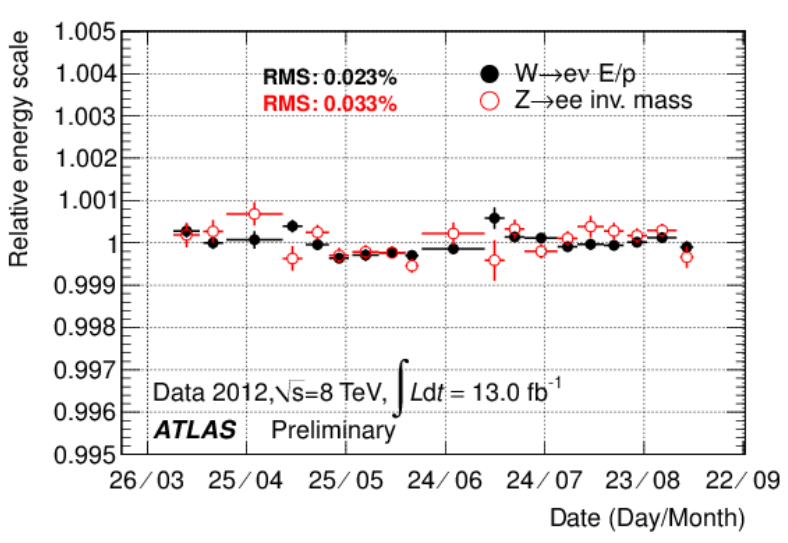

Figure 8: The ATLAS electron energy-scale as a function of time.

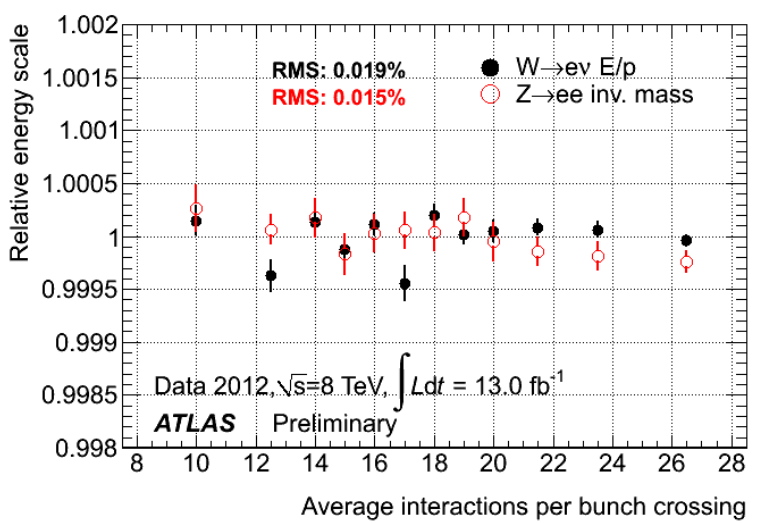

Figure 9: The ATLAS electron energy-scale as a function of the average interactions per bunch crossing.

along $\eta$, the shower-shape variable $\mathrm{R}$, pile-up corrected isolation variables and the amount of hadronic energy deposited behind a calorimeter supercluster (ratio $H / E$ ). Electrons and photons are separated with a track-pointing or a track-veto request, which relies upon an excellent tracker-calorimeter alignment. Efficiencies are computed with a tag-and-probe method using $Z \rightarrow e^{+} e^{-}$events. CMS uses MVA techniques for electron and photon identification, which provide the best performance for Higgs searches. As an example of the performance, Fig. 12 shows the efficiency on data and on a Drell-Yan MC sample for the isolation plus MVA electron selection (loose selection used in $\mathrm{H} \rightarrow \mathrm{ZZ}$ searches) as a function of the number of vertices in the event [21].

\subsection{ATLAS}

The electron identification is based on cuts on shower shape, track quality, hits recorded in the Transition Radiation Tracker [1] and track-cluster matching. Three levels of electron identification cuts are defined with different jet rejection factors: loose (jet rejection $\approx 500$ ), medium (jet rejection $\approx 5000$ ) and tight (jet rejection $\approx 50000$ ). The identification efficiencies are measured in-situ using electrons from $\mathrm{Z} \rightarrow$ ee, $\mathrm{J} / \Psi \rightarrow$ ee and $\mathrm{W} \rightarrow \mathrm{e} v$. The electron identification efficiency only slightly

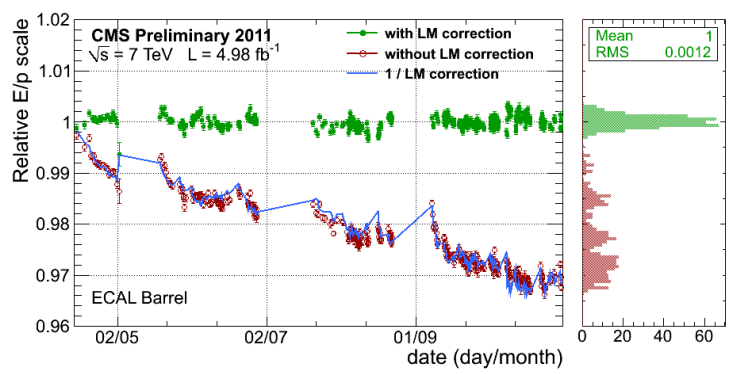

Figure 10: The electron relative energy-scale in the CMS barrel before (red points) and after (green points) laser corrections is shown as a function of time. Each point in the plot is computed from 12000 selected $W$ events with the reconstructed electron located in the barrel. The magnitude of the average transparency correction for each point (averaged over all crystals in the reconstructed electromagnetic clusters) is indicated by the continuous blue line.

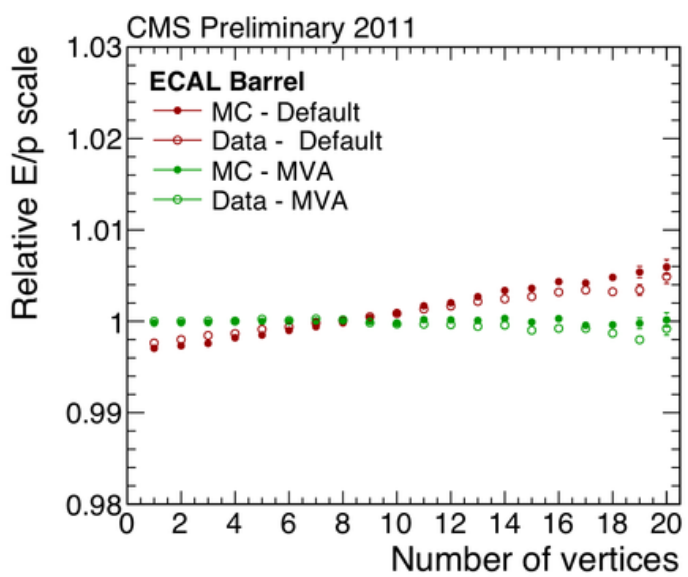

Figure 11: Dependence of the reconstructed energy on the number of reconstructed vertices in CMS events. The default reconstruction of the data (open red circles) and $\mathrm{MC}$ (filled red circles) is compared to MC-driven corrections to the energy based on a multivariate analysis (MVA) of the energy response which includes pileup sensitive global event variables, for the data (open green circles) and MC (filled green circles).

decreases with pile-up as can be seen in Fig. 13 (e.g. decrease of identification efficiency for 18 reconstructed primary vertices compared to no pile-up is only $\approx 2 \%$ ). The main goal of the photon identification is to distinguish direct photons from jets including the case where jets fragment to "single" $\pi^{0}$. ATLAS relies on the high transverse granularity of the first calorimeter layer (see Sec. 1) to reject $\pi^{0}$ candidates with high efficiency. Like the electron efficiencies, the photon efficiencies are measured with data, only a small decrease with increasing number of primary vertices is observed [22]. 


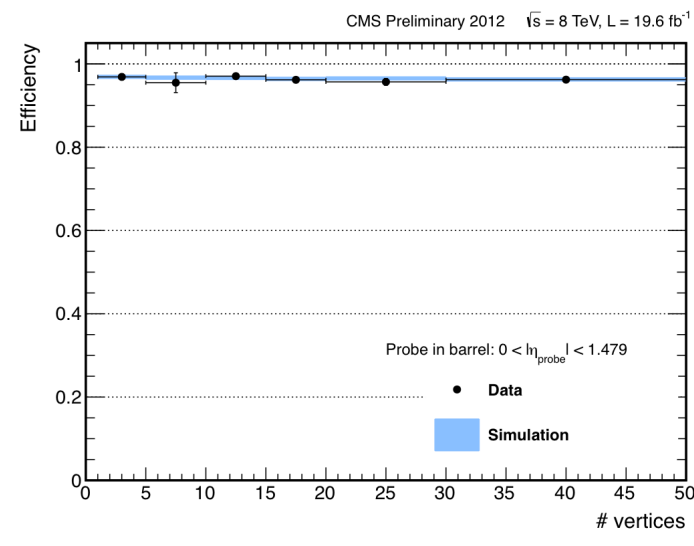

Figure 12: CMS electron efficiency on data and on a DrellYan Monte Carlo sample for the isolation plus the multivariate electron selection as a function of the number of reconstructed vertices in the event.

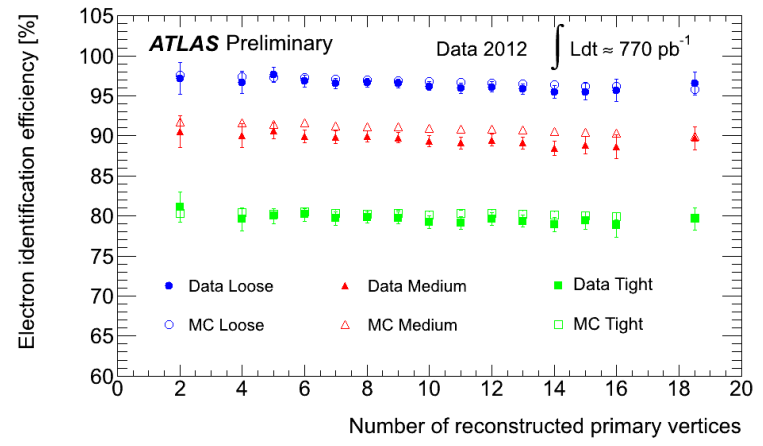

Figure 13: ATLAS electron reconstruction efficiency as a function of the reconstructed number of vertices measured with the so-called "Tag and Probe" method using $\mathrm{Z} \rightarrow$ ee events in data and MC.

\section{Performance of the calorimeter in physics channels}

In this section we want to highlight the role of the electromagnetic calorimeters in the discovery of the Higgs boson ([3] and [4]). We will concentrate on the most relevant decay channel for the electromagnetic calorimeters, which is $\mathrm{H} \rightarrow \gamma \gamma$.

\subsection{ATLAS}

The basic recipe of the Higgs searches in the $\mathrm{H} \rightarrow \gamma \gamma$ decay channel [23] is to select candidates with two photons, reject the jet-jet and $\gamma$-jet backgrounds, reconstruct the invariant mass as precisely as possible and finally optimize the analysis to improve signal significance and statistical power (12 categories, additional kinematic variables). Many of these steps rely entirely on the electromagnetic calorimeter: As already described in previous sections, the high granularity of the first calorimeter layer contributes to the high jet rejection leading to a very pure $\gamma \gamma$ spectrum, the fraction of genuine diphoton events is found to be $75_{-4}^{+3} \%$ [23]. The longitudinal vertex position has a spread of $\approx 5 \mathrm{~cm}$. The longitudinal segmentation of the calorimeter is therefore used to determine event by event the position of the $\mathrm{H} \rightarrow \gamma \gamma$ vertex along the beam line and therefore improve significantly the invariant mass resolution in the case of unconverted photons. The final vertex is chosen with a Neural Network algorithm which combines the photon pointing with, for each reconstructed vertex: the conversion information, the sum of the squared momentum $\sum p_{\mathrm{T}}^{2}$ and the scalar sum of the momentum $\sum p_{\mathrm{T}}$ the tracks associated with each reconstructed vertex, and the difference in azimuthal angle $\Delta \varphi$ between the direction of the vector sum of the tracks momenta and that of the diphoton system. The vertex selection was studied with $\mathrm{Z} \rightarrow$ ee events in data and MC simulation by removing the electron tracks from the events and verifying the efficiency of finding the vertex associated to them. Corrections were applied in order to mimic the Higgs boson signal. The efficiency of finding the primary vertex within $0.3 \mathrm{~mm}$ of the true one is higher than $80 \%$ ([23] and [24]). The resulting numbers of the predicted mass resolution (Gaussian width of Crystal Ball fit $\sigma_{C B}$ and FWHM) for all categories used in [23] are shown in Tab. 1.

\begin{tabular}{ccccccc}
\hline$\sqrt{s}$ & \multicolumn{7}{c}{$8 \mathrm{TeV}$} \\
\hline Category & $\sigma_{\mathrm{CB}}(\mathrm{GeV})$ & FWHM $(\mathrm{GeV})$ & Observed & $N_{S}$ & $N_{B}$ & $N_{S} / N_{B}$ \\
\hline Unconv. central, low $p_{\mathrm{Tt}}$ & 1.47 & 3.45 & 569 & 29 & 538 & 0.053 \\
Unconv. central, high $p_{\mathrm{Tt}}$ & 1.37 & 3.22 & 25 & 4.2 & 25 & 0.168 \\
Unconv. rest, low $p_{\mathrm{Tt}}$ & 1.59 & 3.75 & 2773 & 61 & 2610 & 0.023 \\
Unconv. rest, high $p_{\mathrm{Tt}}$ & 1.52 & 3.59 & 148 & 8.7 & 138 & 0.063 \\
Conv. central, low $p_{\mathrm{Tt}}$ & 1.64 & 3.86 & 446 & 18 & 417 & 0.044 \\
Conv. central, high $p_{\mathrm{Tt}}$ & 1.49 & 3.51 & 18 & 2.8 & 17 & 0.163 \\
Conv. rest, low $p_{\mathrm{Tt}}$ & 1.83 & 4.32 & 2898 & 54 & 2763 & 0.019 \\
Conv. rest, high $p_{\mathrm{Tt}}$ & 1.7 & 4.00 & 144 & 7.4 & 138 & 0.053 \\
Conv. transition & 2.35 & 5.57 & 1872 & 25 & 1825 & 0.014 \\
High Mass two-jet & 1.55 & 3.65 & 47 & 6.8 & 33 & 0.204 \\
Low Mass two-jet & 1.46 & 3.45 & 62 & 4.2 & 45 & 0.093 \\
One-lepton & 1.63 & 3.85 & 18 & 1.7 & 16 & 0.108 \\
\hline Inclusive & 1.64 & 3.87 & 8802 & 223 & 8284 & 0.027 \\
\hline
\end{tabular}

Table 1: ATLAS: Gaussian width of the Crystal Ball function fit $\sigma_{\mathrm{CB}}$ and FWHM of the predicted mass resolution of $\mathrm{H} \rightarrow \gamma \gamma$ for $m_{\mathrm{H}}=126.5 \mathrm{GeV}$ for the different categories used in [23] as well as the number of expected signal events $N_{S}$, the number of background events $N_{B}$ and the ratio $N_{S} / N_{B}$ for $13 \mathrm{fb}^{-1}$ collected at $\sqrt{s}=8 \mathrm{TeV}$.

\begin{tabular}{|c|c|c|c|c|c|}
\hline \multicolumn{6}{|c|}{ Expected signal and estimated background $\left(m_{\mathrm{H}}=125 \mathrm{GeV}\right.$} \\
\hline \multicolumn{2}{|c|}{ Event classes } & \multirow{2}{*}{$\begin{array}{l}\mathrm{N}_{s} \\
17.0\end{array}$} & \multirow{2}{*}{\begin{tabular}{|c|}
$\begin{array}{c}\text { FWHM/2.35 } \\
(\mathrm{GeV})\end{array}$ \\
1.27
\end{tabular}} & \multicolumn{2}{|c|}{$\begin{array}{l}\text { Background } \\
\text { (ev./GeV) }\end{array}$} \\
\hline- & Untagged 0 & & & 22.1 & \pm 0.5 \\
\hline \& & Untagged 1 & 37.8 & 1.39 & 94.3 & \pm 1.0 \\
\hline مै & Untagged 2 & 150.2 & 1.54 & 570.5 & \pm 2.6 \\
\hline$\stackrel{-}{>}$ & Untagged 3 & 159.9 & 2.14 & 1060.9 & \pm 3.5 \\
\hline 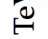 & Dijet tight & 9.2 & 1.50 & 3.4 & \pm 0.2 \\
\hline$\infty$ & Dijet loose & 11.5 & 1.60 & 12.4 & \pm 0.4 \\
\hline & Muon tag & 1.4 & 1.52 & 0.7 & \pm 0.1 \\
\hline & Electron tag & 0.9 & 1.54 & 0.7 & \pm 0.1 \\
\hline & $E_{\mathrm{T}}^{\text {miss }}$ tag & 1.7 & 1.64 & 1.8 & \pm 0.1 \\
\hline
\end{tabular}

Table 2: CMS: Expected signal, predicted mass resolution of $\mathrm{H} \rightarrow \gamma \gamma$ for $m_{\mathrm{H}}=125 \mathrm{GeV}$ and and estimated background events/ $\mathrm{GeV}$ in the signal region for the different categories used in [20]. The numbers refer to a statistics of $19.6 \mathrm{fb}^{-1}$ collected at $\sqrt{s}=8 \mathrm{TeV}$.

\section{2. $C M S$}

The $\mathrm{H} \rightarrow \gamma \gamma$ analysis uses MVA techniques both for photon identification and event classification. It is optimized to exploit 
the varying signal-over-background ratio and mass resolution across the calorimeter [20]. Further classes are defined to identify events from specific production mechanisms requiring the presence of additional physics objects in the final state. Key points for the signal detection are the selection of a clean sample of high $p_{\mathrm{T}}$ photons, the determination of the angle between the photons and their energy measurements. In the diphoton mass range $110-150 \mathrm{GeV}$ the background is dominated by the diphoton continuum, which is predicted by MC to be about $70 \%$ of the total. The photon identification algorithm has been proven to be rather effective. The CMS electromagnetic calorimeter has no longitudinal segmentation and the primary vertex determination relies on an MVA algorithm based on tracks belonging to a vertex combined with the two photon kinematics (it basically uses the sum $\sum p_{\mathrm{T}}^{2}$ of each reconstructed vertex and the two photon system $p_{\mathrm{T}}$ imbalance). If either of the photons converts upstream, the direction of the converted photon tracks can also be used to identify the diphoton production vertex. The efficiency of the vertex-finding algorithm has been measured in high pileup conditions (20 pileup events on average) using $\mathrm{Z} \rightarrow \mu \mu$ events after removal of the muon tracks. The efficiency of finding the primary vertex within $10 \mathrm{~mm}$ from the true position for a Higgs boson of mass $120 \mathrm{GeV}$, integrated over its $p_{\mathrm{T}}$ spectrum, is $80 \%$ [20]. The mass resolutions predicted for the different event categories, as well as the expected signal and background for a higgs mass of $125 \mathrm{GeV}$, are reported in Tab. 2 .

\subsection{Comparison}

It is not straightforward to compare CMS and ATLAS performance since the categories don't correspond exactly. In addition, ATLAS uses a cut-based classification whereas CMS uses MVA categories. However, we will try in the following a very basic and approximate comparison: CMS MVA category untagged 0 contains boosted events, has the best mass resolution and signal-to-background ratio and can be compared to the ATLAS unconverted and converted central high $p_{\mathrm{T}}$. CMS MVA category untagged 1 roughly corresponds to ATLAS unconverted central low $p_{\mathrm{T}}$. The remaining ATLAS categories can be related with the CMS MVA untagged 2 and untagged 3 categories.

A comparison of the results of ATLAS and CMS in terms of performance of the calorimeters, looking at the $\mathrm{H} \rightarrow \gamma \gamma$, can be attempted using the numbers reported in Tab. 1 and Tab. 2 using the FWHM but taking into account the different statistics and the fact that the number of signal and background events quoted in the ATLAS table is counted in a window containing the $90 \%$ of the signal $(\sim 1.645 \sigma)$. For example, CMS category untagged 0 has a FWHM of $2.98 \mathrm{GeV}$, which can be roughly compared with the FWHM of the ATLAS unconverted central high $p_{\mathrm{T}}$ with an FWHM of $3.22 \mathrm{GeV}$ and converted central high $p_{\mathrm{T}}$ with an FWHM of $3.51 \mathrm{GeV}$. The slightly better mass resolution obtained by CMS is expected due to the better energy resolution of the calorimeter; however, the difference for unconverted photons is rather small due to the more precise vertex identification possible with ATLAS due to photon pointing. Comparing the number of signal and background events for these categories shows that the number of signal events $N_{S}$ and $N_{B}$ and also their ratio is comparable between ATLAS and CMS. Even though comparing these best categories shows the better mass resolution of the CMS calorimeter, the spread of resolutions for the different categories is larger than in ATLAS resulting in the same inclusive mass resolution in ATLAS and CMS. This together with different background rejection capabilities of the two experiments yields an expected signal significance at the same level.

\section{Upgrade studies and plans}

The expected instantaneous LHC luminosity after 2018 is $\mathrm{L}_{\text {inst }} \approx 2-3 \cdot 10^{34} \mathrm{~cm}^{-2} \mathrm{~s}^{-1}$ corresponding to an average number of interactions per bunch crossing (pile-up) of $\langle\mu\rangle=55-81$. For high luminosity (HL) LHC, planned for the time after 2022 an instantenous luminosity of up to $\mathrm{L}_{\text {inst }} \approx 5-7 \cdot 10^{34} \mathrm{~cm}^{-2} \mathrm{~s}^{-1}$ with an average pileup of $\langle\mu\rangle=140$ is foreseen.

\subsection{ATLAS}

The LAr calorimeter is expected to be fully operational with similar performance as now at luminosities up to $\mathrm{L}_{\mathrm{inst}} \approx 3$. $10^{34} \mathrm{~cm}^{-2} \mathrm{~s}^{-1}$. However, the calorimeter level 1 trigger will suffer from both, high pile-up and high instantaneous luminosity. Since the level 1 trigger rate will be limited to $100 \mathrm{kHz}$, in order to avoid a significant raise of level 1 trigger thresholds for electrons/photons, jets, taus and missing transverse energy better rejection against background will be necessary. The LAr calorimeter trigger upgrade therefore aims in increasing the granularity of the level 1 calorimeter trigger to improve rejection against jets in the electromagnetic triggers, improve the turn-on curves for jets and improve missing transverse energy resolution despite the high pile-up. To achieve the higher granularity calorimeter trigger the analog level 1 trigger tower signals will be replaced by digital transmission of the signals of "super-cells" obtained by summing 4-8 read-out cells in one layer (in the barrel there will be 10 "super-cells" per analog trigger tower). In addition to the energies of the higher granularity super-cells, the level 1 trigger will also incorporate information on lateral and longitudinal shower shapes into the trigger decision [25].

For the HL-LHC it is planned to change the read-out electronics of the LAr calorimeter to be able to cope with a higher latency of the trigger and to use the full granularity calorimeter information already in a low-level trigger decision [26].

\section{2. $C M S$}

CMS was designed for $\mathrm{L}_{\text {inst }}=10^{34} \mathrm{~cm}^{-2} \mathrm{~s}^{-1}$ (average of 25 interactions per bunch crossing) and 10 years running for a total integrated luminosity of $500 \mathrm{fb}^{-1}$. The corresponding gamma irradiation rates are $0.3 \mathrm{~Gy} / \mathrm{h}$ in the barrel and $6.5 \mathrm{~Gy} / \mathrm{h}$ in the end-caps at $\eta=2.6$. In the same regions of barrel and endcaps fluences of $4 \cdot 10^{11}$ protons $/ \mathrm{cm}^{2}$ and $3 \cdot 10^{13}$ protons $/ \mathrm{cm}^{2}$ are expected. The calorimeter is expected to maintain its performance even with the doubling of the design luminosity foreseen in the years 2019-2021. Coming to the HL-LHC phase, the severe irradiation and the high pileup may cause a deterioration 
of the performance of the calorimeter particularly in the endcaps region. The irradiation levels will be approximately ten times higher than the design values. In particular, hadron damage creates clusters of defects that cause a light transmission loss in the crystals. This damage is permanent and cumulative at room temperature. Other effects include an increase of APD dark current (increase of noise in the barrel) due to the neutron fluence and an increase of spike rate in the barrel. Detailed studies are ongoing to understand (and possibly mitigate) any degradation of the physics performance of the calorimeter, due to $\gamma$ and hadron irradiation.

\section{Conclusions}

The electromagnetic calorimeters of ATLAS and CMS have been described in detail. Their performance during the first three years of LHC operation has been discussed and compared. It has been shown, that the electromagnetic calorimeters of ATLAS and CMS both perform as expected and deliver a very high fraction of good quality data for physics analysis. The electromagnetic calorimeters played an important role in the discovery of the Higgs boson.

\section{Acknowledgements}

We thank CERN for the very successful operation of the LHC, as well as the support staff from our institutions without whom ATLAS and CMS could not be operated efficiently.

\section{References}

[1] ATLAS Collaboration, The ATLAS experiment at the CERN LHC, J. Instrum., 3 S08003 (2008)

[2] CMS Collaboration, The CMS experiment at the CERN LHC, J. Instrum 3 S08004 (2008)

[3] CMS Collaboration, Phys. Lett. B716 (2012)

[4] ATLAS Collaboration, Phys. Lett. B716 (2012)

[5] ATLAS Collaboration, Liquid Argon Calorimeter Technical Design Report, CERN/LHCC/96-041 (1996)

[6] CMS Collaboration, The Electromagnetic Calorimeter Technical Design Report, CERN/LHCC/97-33 (1997)

[7] S. Baccaro et al., Nucl. Instrum. Meth. A 459 (2001) 278; E. Auffray et al., Nucl. Instrum. Meth. A 456 (2001) 325; E. Auffray et al., Nucl. Instrum. Meth. A 523 (2004) 355.

[8] P. Adzic et al., JINST 5 (2010) P03010

[9] M. Anfreville et al., Nucl. Instrum. Met., A594 (2008) 292

[10] CMS Collaboration, JINST 5 (2010) T03010

[11] P.Adzic, et al., Eur. Phys. J. C44 (s02) (2006) 1.

[12] A. Bartoloni et al., Nucl. Instrum. Meth. A582 (2007) 462
[13] B. Aubert et al., Nucl. Instrum. Meth., A 558 (2006)

[14] M. Aleksa et al., J. Instrum., 3 P06002 (2008)

[15] D. A. Petyt, Nucl. Instrum. Meth. A 695 (2011) 293

[16] M. Aharrouche et al., Nucl. Instrum. Meth., A 582 (2007)

[17] ATLAS Collaboration, Eur. Phys. J. C 72 (2012) 1909

[18] CMS Collaboration, EGM-11-001, to be submitted to JINST

[19] P. Adzic et al., JINST 2 (2007) P04004

[20] CMS Collaboration, CMS-PAS-HIG-2013-001

[21] CMS Collaboration, CMS DP-2013/003

[22] ATLAS Collaboration, ATLAS-CONF-2012-123 (2012)

[23] ATLAS Collaboration, ATLAS-CONF-2012-168 (2012)

[24] ATLAS Collaboration, ATLAS-CONF-2012-091 (2012)

[25] ATLAS Collaboration, CERN-LHCC-2011-012 (2011)

[26] ATLAS Collaboration, CERN-LHCC-2012-022 (2012) 